\title{
Spontaneous spectral phase conjugation for coincident frequency entanglement
}

\author{
Mankei Tsang and Demetri Psaltis \\ Department of Electrical Engineering, California Institute of Technology, Pasadena, California 91125, USA
}

(Received 19 October 2004; revised manuscript received 30 November 2004; published 11 April 2005)

\begin{abstract}
Spontaneous parametric processes pumped transversely with short pulses are studied under a unified framework, which proves that such processes can efficiently generate entangled photon pairs with time anticorrelation and frequency correlation. Improvements upon previously proposed schemes can be made by the use of quasi-phase-matching, four-wave mixing, and cross-phase-modulation compensation. The use of frequencycorrelated photons in the Hung-Ou-Mandel interferometer is studied.
\end{abstract}

DOI: $10.1103 /$ PhysRevA.71.043806

PACS number(s): 42.65.Lm, 42.50.Dv

It has recently been proven that if the three-wave-mixing (TWM) or four-wave-mixing (FWM) parametric process is transversely pumped with short pulses in a long and thin nonlinear medium, parametric amplification can be performed, with time reversal and spectral phase conjugation (SPC) $[1,2]$. The correspondence between classical parametric amplification and quantum entanglement makes one wonder if spontaneous SPC can perform the opposite of what its continuous-wave-pumped counterpart does, and realize time anticorrelation or frequency correlation. This distinct kind of entanglement is useful for applications such as quantumenhanced position and clock synchronization [3] and oneway autocompensating quantum cryptography [4]. Various implementations of such entanglement have been suggested $[5,6]$, and the scheme proposed by Walton et al. [6] looks intriguingly similar to the TWM scheme for SPC [1]. On the other hand, while TWM is traditionally the preferred method to generate entangled photons, recent experimental progress on entangled photon sources using third-order nonlinear processes $[7,8]$ makes FWM a promising candidate for such a task. In this paper, we prove that spontaneous SPC, by either TWM or FWM, can indeed efficiently generate timeanticorrelated and frequency-correlated photon pairs.

Our proposed schemes have several key improvements over that in Ref. [6], and make coincident frequency entanglement much more realizable. First, it is unclear in Ref. [6] how phase matching should be achieved. We propose the incorporation of quasi-phase-matching to satisfy the requirement. Second, we suggest an alternative FWM scheme, which can be more efficient with focused femtosecond pump beams. Third, for good efficiency, cross-phase modulation due to the strong pump becomes a large parasitic effect for both schemes. We introduce the use of pump phase modulation to compensate for cross-phase modulation. We also perform an in-depth Heisenberg analysis of the spontaneous SPC schemes, predicting that a high gain is possible with current technology. The high gain enables the generation of large-photon-number frequency-correlated states, which are interesting for their use in nonlocal-dispersion cancellation and noise-reduction experiments [9].

Lastly we investigate the use of frequency-correlated photons generated by our proposed schemes in the Hong-OuMandel (HOM) interferometer [10], subject to temporal delays, dispersion, frequency shifts, and temporal phase modulation. Quantum-dispersion cancellation in the HOM interferometer with such photons has been studied [10-13], but relatively little attention is given to the distinguishability introduced by phase modulation in the time domain, for example, via the optical Kerr effect, which can be useful in quantum nondemolition measurements [14]. Reference [11] studies the effect of frequency shifts on frequency-correlated photons, but only in a highly idealized case. Using the formalism developed for our schemes, we first review the HOM dip effect introduced by time delays and dispersion for completeness, and then study the nonlocal temporal phasecancellation properties of the entangled photons.

The spontaneous SPC scheme by TWM is sketched in Fig. 1, and the FWM scheme in Fig. 2. For the TWM scheme, the interaction Hamiltonian is

$$
\hat{H} \propto w \int d x \int d z \chi^{(2)} f(z) E_{p}^{(+)} E_{s}^{(-)} E_{i}^{(-)}+\text {H.c. },
$$

where $w$ is the width of the nonlinear medium in the $y$ dimension, $\chi^{(2)}$ is the second-order nonlinear susceptibility, $f(z)$ is the pump-beam profile, $E^{(+)}$and $E^{(-)}$are the positivefrequency and negative-frequency electric field operators, respectively, and the subscripts $p, s$, and $i$ denote pump, signal, and idler, respectively. If the pump is assumed to be classical, the electric field operators can be written in terms of envelopes $\quad E_{p}^{(+)} \propto A_{p}\left(t+x / v_{x}\right) \exp \left(-i 2 \omega_{0} t-i k_{x} x\right), \quad E_{s}^{(-)} \propto \hat{A}_{s}^{\dagger}(t$ $-z / v) \exp \left(i \omega_{0} t-i k_{z} z\right)$, and $E_{i}^{(-)} \propto \hat{A}_{i}^{\dagger}(t+z / v) \exp \left(i \omega_{0} t+i k_{z} z\right)$, where $k_{x}$ is the pump carrier-wave vector, and $v$ and $v_{x}$ are the group velocities in $z$ and $x$, respectively. Unlike the scheme in Ref. [5], spontaneous SPC places no restriction on the material dispersion properties as long as the signal and idler are the same but counterpropagating modes. Otherwise the pump beam(s) can be slightly tilted in the $z$ direction to compensate fo the signal-idler phase mismatch. The interaction Hamiltonian then becomes

$$
\begin{aligned}
\hat{H} \propto & \int_{-d / 2}^{d / 2} d x \int d z \chi^{(2)} f(z) A_{p}\left(t+\frac{x}{v_{x}}\right) \hat{A}_{s}^{\dagger}\left(t-\frac{z}{v}\right) \hat{A}_{i}^{\dagger}\left(t+\frac{z}{v}\right) \\
& \times \exp \left[-i\left(k_{x}-\frac{2 \pi}{\Lambda}\right) x\right]+\text { H.c. }
\end{aligned}
$$




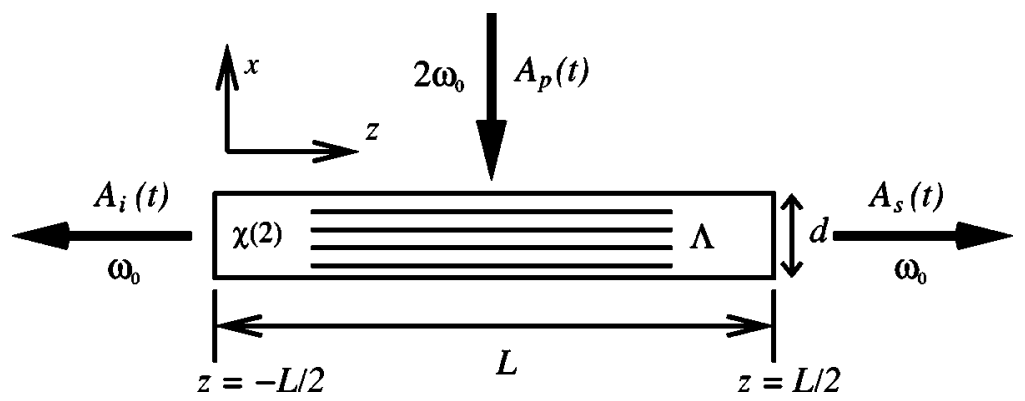

FIG. 1. Spontaneous SPC by TWM.

where $\Lambda$ is the $\chi^{(2)}$ grating period. Reference [15] assumes that the transverse dimension $d$ is small enough so that detuning due to $k_{x}$ can be ignored. However, for a realizable setup, $k_{x}$ is usually on the order of $1 /(1 \mu \mathrm{m})$, while $d$ is on the order of micrometers for a waveguide. Hence in most cases $k_{x}$ should not be ignored, and quasi-phase-matching, not mentioned in Ref. [6], is in fact needed. The submicrometer $\chi^{(2)}$ grating period required can be fabricated, for example, in a GaAs/ $/ \mathrm{Al}_{x} \mathrm{Ga}_{1-x} \mathrm{As}$ heterostructure [16]. To avoid space-time coupling, the spatial pulse width of the pump, on the order of $100 \mu \mathrm{m}$ for a femtosecond pulse, should be much larger than $d \sim 10 \mu \mathrm{m}$, so that the $x$ dependence of $A_{p}$ can be neglected. $\hat{H}$ is then given by

$$
\hat{H} \propto w d \int d z \chi^{(2)} f(z) A_{p}(t) \hat{A}_{s}^{\dagger}\left(t-\frac{z}{v}\right) \hat{A}_{i}^{\dagger}\left(t+\frac{z}{v}\right)+\text { H.c. }
$$

The FWM process, on the other hand, is automatically phase matched due to the counterpropagating pump pulses. The interaction Hamiltonian is

$$
\hat{H} \propto w \int d x \int d z \chi^{(3)} f(z) E_{p}^{(+)} E_{q}^{(+)} E_{s}^{(-)} E_{i}^{(-)}+\text {H.c. }
$$

With similar assumptions as in the TWM scheme, the interaction Hamiltonian is given by

$$
\hat{H} \propto w d \int d z \chi^{(3)} f(z) A_{p}(t) A_{q}(t) \hat{A}_{s}^{\dagger}\left(t-\frac{z}{v}\right) \hat{A}_{i}^{\dagger}\left(t+\frac{z}{v}\right)+\text { H.c. },
$$

which is almost identical to that of the TWM scheme. Hence one can analyze both schemes in a unified framework, with the general Hamiltonian

$$
\hat{H} \propto \int d z f(z) g(t) \hat{A}_{s}^{\dagger}\left(t-\frac{z}{v}\right) \hat{A}_{i}^{\dagger}\left(t+\frac{z}{v}\right)+\text { H.c. }
$$

where $g(t) \propto \chi^{(2)} A_{p}(t)$ for the TWM scheme and $g(t)$ $\propto \chi^{(3)} A_{p}(t) A_{q}(t)$ for the FWM scheme. To the first order, the wave function is given by [6]

$$
\begin{gathered}
\left|\psi^{\prime}\right\rangle \propto \int d t \hat{H}|0\rangle \\
\propto \int d t \int d z g(t) f(z) \hat{A}_{s}^{\dagger}\left(t-\frac{z}{v}\right) \hat{A}_{i}^{\dagger}\left(t+\frac{z}{v}\right)|0\rangle \\
\propto \int d \omega_{s} \int d \omega_{i} \tilde{f}\left(\frac{\omega_{s}-\omega_{i}}{v}\right) \tilde{g}\left(\omega_{s}+\omega_{i}\right) \hat{a}_{s}^{\dagger}\left(\omega_{s}\right) \hat{a}_{i}^{\dagger}\left(\omega_{i}\right)|0\rangle,
\end{gathered}
$$

where $\tilde{f}, \widetilde{g}$, and $\hat{a}_{s, i}^{\dagger}$ are Fourier transforms of $f, g$, and $\hat{A}_{s, i}^{\dagger}$, respectively. In the time domain, if the width of $g(t)$ is much narrower than the width of $f(z)$ divided by $v, g(t)$ can sample the integrand in Eq. (8), say at $t=0$. Equivalently in the frequency domain $\tilde{f}$ can sample the integrand at $\omega_{s}=\omega_{i}$ in Eq. (9). The wave function becomes

$$
\begin{aligned}
& \left|\psi^{\prime}\right\rangle \propto \int d t^{\prime} g\left(t^{\prime}\right) \int d t f(v t) \hat{A}_{s}^{\dagger}(-t) \hat{A}_{i}^{\dagger}(t)|0\rangle \\
& \propto \int d \omega^{\prime} \tilde{f}\left(\frac{\omega^{\prime}}{v}\right) \int d \omega \tilde{g}(2 \omega) \hat{a}_{s}^{\dagger}(\omega) \hat{a}_{i}^{\dagger}(\omega)|0\rangle .
\end{aligned}
$$

The generated photon pair therefore possesses quantum time anticorrelation and frequency correlation. In summary, for optimal entanglement, the assumption

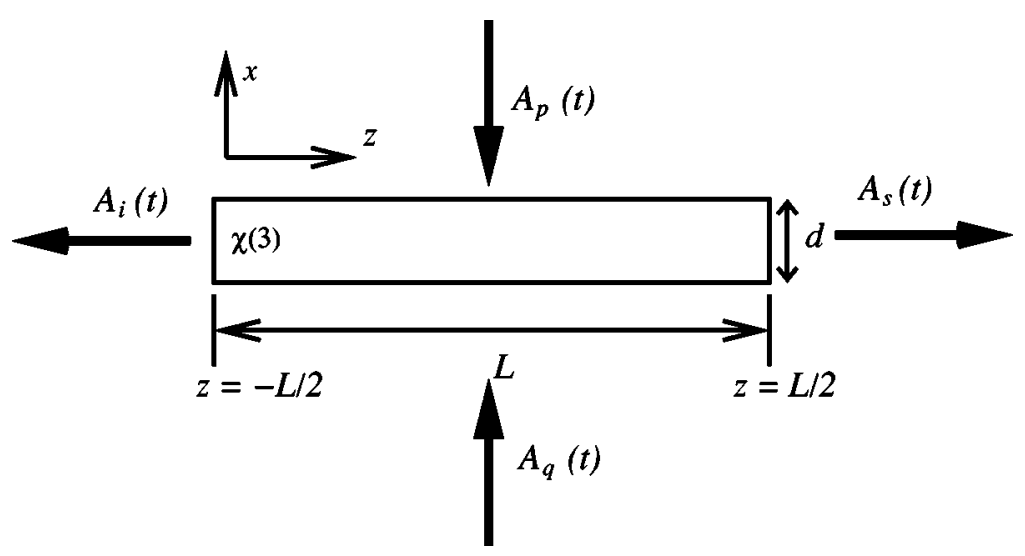

FIG. 2. Spontaneous SPC by FWM. 


$$
\frac{L}{v} \gg \text { width of } g(t) \gg \frac{d}{v_{x}}
$$

should be satisfied.

The efficiency of spontaneous SPC is best studied in the Heisenberg picture. The coupled-operator equations, assuming classical undepleted pumps, are given by

$$
\begin{gathered}
\left(v \frac{\partial}{\partial z}+\frac{\partial}{\partial t}\right) \hat{A}_{s}=i g(t) \hat{A}_{i}^{\dagger}+i c(t) \hat{A}_{s}, \\
\left(-v \frac{\partial}{\partial z}+\frac{\partial}{\partial t}\right) \hat{A}_{i}^{\dagger}=-i g^{*}(t) \hat{A}_{s}-i c(t) \hat{A}_{i}^{\dagger},
\end{gathered}
$$

where

$$
g(t)=\frac{\omega_{0} v \chi^{(2)}}{2 c n_{0}} A_{p}(t)
$$

for the TWM scheme and

$$
g(t)=\frac{3 \omega_{0} v \chi^{(3)}}{4 c n_{0}} A_{p}(t) A_{q}(t)
$$

for the FWM scheme.

$$
c(t)=\frac{3 \omega_{0} v \chi^{(3)}}{4 c n_{0}}\left(\left|A_{p}\right|^{2}+\left|A_{q}\right|^{2}\right)
$$

is the cross-phase-modulation term, which acts as a timedependent detuning factor. Cross-phase-modulation is always present in the FWM scheme, while it exists in the form of competing third-order nonlinearity in the TWM scheme. Equations (13) and (14) can be solved in the same manner as in the classical SPC analysis [1,2]. The temporary detuning due to cross-phase-modulation can be compensated if $g(t)$ is also appropriately detuned. Quantitatively, the phase of $g(t)$ should be modulated as [2]

$$
\theta(t)=\theta_{0}+2 \int_{-\infty}^{t} d t^{\prime} c\left(t^{\prime}\right)
$$

$\theta(t)$ can be approximated by a linear temporal phase, or a center-frequency shift of $g(t)$ [2]. In other words, for spontaneous SPC under the cross-phase-modulation effect, the generation of photon pairs will actually be most efficient at a center frequency different from the center pump frequency in the FWM scheme, or from half the center second-harmonic pump frequency in the TWM scheme. This is analogous to the phenomenon of sideband gain in continous-wave FWM, although now the signal and idler spectra should coincide with each other. This feature is actually desirable for the FWM scheme, since it is easier to separate the scattered pump from the weak signal and idler by spectral filtering.

The average number of photons in each mode is

$$
\begin{aligned}
& n_{s}=C^{2} n_{s 0}+S^{2}\left(n_{i 0}+1\right), \\
& n_{i}=C^{2} n_{i 0}+S^{2}\left(n_{s 0}+1\right),
\end{aligned}
$$

where $C \equiv \cosh \left[\int d t|g(t)|\right], S \equiv \sinh \left[\int d t|g(t)|\right], n_{s 0}$ is the initial signal photon number, and $n_{i 0}$ is the initial idler photon number. The number of photon pairs spontaneously generated in each wave-mixing event is therefore $S^{2}$, and the conversion efficiency, defined as the energy of the generated photons divided by the energy of the pump photons, is

$$
\Gamma=\frac{2 S^{2} \hbar \omega_{0}}{\mathcal{E}_{p}},
$$

where $\mathcal{E}_{p}$ is the total pump energy. The FWM scheme is more efficient than the TWM scheme when $g(t)$ of the former is larger, or, all else being equal,

$$
\frac{\chi^{(3)}}{\left(n_{0}^{(3)}\right)^{3}}\left(\frac{\mathcal{E}_{p}}{\epsilon_{0} c L w T_{p}}\right)^{1 / 2} \gtrsim \frac{\chi^{(2)}}{\left(n_{0}^{(2)}\right)^{5 / 2}},
$$

where $T_{p}$ is the pump pulse width.

For example, polydiacetylene, a conjugated polymer, has a $\chi^{(3)} \sim 10^{-18} \mathrm{~m}^{2} / \mathrm{V}^{2}$ and $n_{0}^{(3)} \sim 2$, while a GaAs/Al $\mathrm{Ga}_{x} \mathrm{Ga}_{1-x} \mathrm{As}$ heterostructure has a $\chi^{(2)} \sim 10^{10} \mathrm{~m} / \mathrm{V}$ and $n_{0}^{(2)} \sim 4$. For a focused femtosecond pump beam, say $L \sim 2 \mathrm{~mm}, w \sim 5 \mu \mathrm{m}$, $T_{p} \sim 100 \mathrm{fs}$, the FWM scheme is more efficient when $\mathcal{E}_{p}$ is approximately larger than $1 \mathrm{~nJ}$. Ti:sapphire laser systems can achieve a pulse energy of $1 \mathrm{~mJ}$ or more, so the FWM scheme can be orders of magnitude more efficient. The FWM scheme also has the advantage of automatic phase matching as well as having pump pulses near the fundamental frequency, thus eliminating the need of quasi-phase-matching and a second-harmonic source in an experiment. That said, the necessity of synchronizing two short pump pulses in the FWM scheme may undermine its robustness, while the TWM scheme may be more efficient for certain parameters and it is relatively easier to filter out the scattered secondharmonic pump from the signal and idler. For the parameters above, the signal and idler gain $S^{2}$ can achieve $100 \%$ for a pump pulse energy $\sim 10 \mathrm{~nJ}$ with the FWM scheme and $\sim 2 \mu \mathrm{J}$ with the TWM scheme. For such a relatively high gain the wave function can have higher-order terms [17], $|\psi\rangle \propto \sum_{n=0}^{\infty} T^{n}|n\rangle_{s}|n\rangle_{i}$, where $T=S / C$, and the weights of largephoton-number states $T^{2 n}$ with $n>1$ become appreciable when $S^{2}$ approaches unity. Amplification of coincident frequency entanglement [17] also becomes possible.

In the HOM interferometry, variable delays are introduced to the signal and idler photons, which then pass through a 50-50 beam splitter and finally the coincidence rate of the two output ports is measured [10]. For simplicity we also assume that the distances from the two detectors to the beam splitter are the same. We start with the more general wave function in Eq. (8). The electric field operators of the two outputs are given by

$$
\hat{A}_{1,2}(t) \propto \hat{A}_{s, i}\left( \pm L_{s, i}, t\right)+i \hat{A}_{i, s}\left(\mp L_{i, s}, t\right),
$$

where $L_{s, i}$ are the distances traveled by the signal and the idler from $z=0$ to the detectors, respectively. $L_{i}$ has a negative sign in front because the idler travels backward. The coincidence rate is given by $[12,13]$

$$
P_{c} \propto \int d t_{1} \int d t_{2}\left\langle\psi^{\prime}\left|\hat{A}_{1}^{\dagger}\left(t_{1}\right) \hat{A}_{2}^{\dagger}\left(t_{2}\right) \hat{A}_{1}\left(t_{1}\right) \hat{A}_{2}\left(t_{2}\right)\right| \psi^{\prime}\right\rangle
$$




$$
\begin{aligned}
& \propto \int d t_{+}\left|g\left(t_{+}-\frac{L_{+}}{v}\right)\right|{ }^{2}\left[\int d t_{-}\left|f\left(L_{-}-v t_{-}\right)\right|^{2}\right. \\
& \left.-\operatorname{Re} \int d t_{-} f^{*}\left(L_{-}-v t_{-}\right) f\left(L_{-}+v t_{-}\right)\right],
\end{aligned}
$$

where $t_{+}=\left(t_{1}+t_{2}\right) / 2, L_{+}=\left(L_{s}+L_{i}\right) / 2, t_{-}=\left(t_{1}-t_{2}\right) / 2$, and $L_{-}$ $=\left(L_{s}-L_{i}\right) / 2$ is half the signal-idler path difference. The shape of the HOM dip with respect to the path difference $L_{s}-L_{i}$ is given by the last term of Eq. (25), which has a width on the order of $L$, the width of the pump-beam profile $f(z)$. Reference [11] predicts that $P_{c}$ is identically zero for all $L_{-}$ with perfectly frequency-correlated photons, which is simply a special case when $L \rightarrow \infty$.

Steinberg et al. predicted [18] and demonstrated [19] even-order dispersion cancellation in the HOM interferometer with frequency-anticorrelated photons. With frequencycorrelated photons, intuition then suggests that one can obtain nonlocal cancellation of dispersion of all orders, which is proven in Ref. [12]. To see how this effect manifests itself in our schemes, we shall start with the general wave function in the frequency domain, Eq. (9), and apply spectral phase to the operators just as in Ref. [18]. The coincidence rate can be expressed in terms of frequency-domain operators as [18]

$$
\begin{aligned}
P_{c} & \propto \int d \omega_{1} \int d \omega_{2}\left\langle\psi^{\prime}\left|\hat{a}_{1}^{\dagger}\left(\omega_{1}\right) \hat{a}_{2}^{\dagger}\left(\omega_{2}\right) \hat{a}_{1}\left(\omega_{1}\right) \hat{a}_{2}\left(\omega_{2}\right)\right| \psi^{\prime}\right\rangle \\
\propto & \int d \omega_{1} \int d \omega_{2}\left|\widetilde{g}\left(\omega_{1}+\omega_{2}\right)\right|^{2}\left\{\left|\tilde{f}\left(\frac{\omega_{1}-\omega_{2}}{v}\right)\right|^{2}\right. \\
& -\operatorname{Re}\left[\tilde { f } ( \frac { \omega _ { 1 } - \omega _ { 2 } } { v } ) \tilde { f } ^ { * } ( \frac { \omega _ { 2 } - \omega _ { 1 } } { v } ) \operatorname { e x p } \left\{i \left[k_{s}\left(\omega_{1}\right)\right.\right.\right. \\
& \left.\left.\left.\left.-k_{s}\left(\omega_{2}\right)\right] L_{s}-i\left[k_{i}\left(\omega_{1}\right)-k_{i}\left(\omega_{2}\right)\right] L_{i}\right\}\right]\right\},
\end{aligned}
$$

where $k_{s}$ and $k_{i}$ are the dispersive propagation constants of the signal and idler, respectively. The exponential term in Eq. (27) characterizes the nonlocal dispersion cancellation. If the photons have perfect frequency anticorrelation, $\widetilde{g}$ is infinitely sharp, $\omega_{1}=-\omega_{2}$, and we recover the even-order dispersion cancellation results in Ref. [18]. If $\tilde{f}$ is infinitely sharp, $\omega_{1}$ $=\omega_{2}$, the exponential term evalulates to 1 and $P_{c}=0$ for dispersion of all orders. If $\tilde{f}$ is not infinitely sharp and dispersion is due only to time delay, the result in Eq. (25) is recovered.

Frequency shifts can be introduced to the signal and the idler by, for example, moving mirrors or acousto-optic modulators via the Doppler effect. Opposite Doppler shifts can also occur to the counterpropagating photons if the source frame is moving along $z$ relative to the detection frame. We can then rewrite $\hat{A}_{1}$ and $\hat{A}_{2}$,

$$
\hat{A}_{1,2}(t) \propto \exp \left(-i \delta \omega_{s, i} t\right) \hat{A}_{s, i}+i \exp \left(-i \delta \omega_{i, s} t\right) \hat{A}_{i, s},
$$

where $\delta \omega_{s}$ is the signal frequency shift and $\delta \omega_{i}$ is the idler frequency shift. For intuitiveness we assume that the path delays for both photons are the same, or $L_{s}=L_{i}=L_{+} . P_{c}$ becomes

$$
\begin{aligned}
P_{c} \propto \int d t_{+}\left|g\left(t_{+}-\frac{L_{+}}{v}\right)\right|^{2}\left(\int d t_{-}\left|f\left(-v t_{-}\right)\right|^{2}\right. \\
\left.-\operatorname{Re} \int d t_{-} f^{*}\left(-v t_{-}\right) f\left(v t_{-}\right) \exp \left[2 i\left(\delta \omega_{s}-\delta \omega_{i}\right) t_{-}\right]\right) .
\end{aligned}
$$

The last term of Eq. (29), which is the Fourier transform of $|f(v t)|^{2}$ if $f(v t)$ is even, characterizes the HOM dip with respect to the frequency difference $\delta \omega_{s}-\delta \omega_{i}$. The width of the dip is on the order of $v / L$, for which the infinitely sharp dip predicted by Ref. [11] is again a special case when $L \rightarrow \infty$.

The coincidence rate depends on the time-domain operators in Eq. (24) in the same way as the expression in Eq. (26) depends on frequency-domain operators,

$$
\begin{aligned}
P_{c} & \propto \int d t_{1} \int d t_{2}\left\langle\psi^{\prime}\left|\hat{A}_{1}^{\dagger}\left(t_{1}\right) \hat{A}_{2}^{\dagger}\left(t_{2}\right) \hat{A}_{1}\left(t_{1}\right) \hat{A}_{2}\left(t_{2}\right)\right| \psi^{\prime}\right\rangle \\
& \propto \int d \omega_{1} \int d \omega_{2}\left\langle\psi^{\prime}\left|\hat{a}_{1}^{\dagger}\left(\omega_{1}\right) \hat{a}_{2}^{\dagger}\left(\omega_{2}\right) \hat{a}_{1}\left(\omega_{1}\right) \hat{a}_{2}\left(\omega_{2}\right)\right| \psi^{\prime}\right\rangle .
\end{aligned}
$$

Hence the coincidence rate obeys a kind of Parseval's relation, and the frequency-domain results can be directly applied to the time domain, if we replace frequency-domain operators with time-domain operators, frequency anticorrelation with time anticorrelation, frequency correlation with time correlation, and spectral phase modulation (dispersion) with temporal phase modulation.

One can introduce temporal phase modulation to the photons by a Doppler shift as mentioned above, or by crossphase-modulation via a classical pulse in a Kerr medium if a more complex phase profile is desired. Given the Parseval relation for coincidence, we then expect the HOM interferometry results with frequency-anticorrelated photons subject to dispersion to be functionally the same as the results with time-anticorrelated photons subject to temporal phase modulation. The coincidence rate is

$$
P_{c} \propto \int d t|f(v t)|^{2}\left\{1-\cos \left[\phi_{s}(t)-\phi_{s}(-t)-\phi_{i}(t)+\phi_{i}(-t)\right]\right\},
$$

where $\phi_{s, i}$ are the temporal phases introduced to the signal and idler photons. The even component of the temporal phase is canceled, as expected. For a linear temporal phase, the result in Eq. (29) is recovered. On the other hand, timecorrelated photons are capable of complete nonlocal temporal phase cancellation. An analogy with classical nonlinearity compensation by different phase conjugation schemes can clearly be made; whereas midway SPC can compensate only for the elastic component of the Kerr effect, midway temporal phase conjugation can compensate for both the instantaneous and delayed Kerr effects [20]. 
The Parseval relation for coincidence of course holds for any kind of interferometer, so any result with other interoferometers obtained with frequency-anticorrelated photons subject to dispersion can be applied directly to time- anticorrelated photons subject to temporal phase modulation. For instance, the extensive study of Mach-Zehnder interferometry with frequency anticorrelated photons in Ref. [11] can be utilized just as well in the time domain.
[1] M. Tsang and D. Psaltis, Opt. Commun. 242, 659 (2004).

[2] M. Tsang and D. Psaltis, Opt. Express 12, 2207 (2004).

[3] V. Giovannetti, S. Lloyd, and L. Maccone, Nature (London) 412, 417 (2001).

[4] Z. D. Walton, A. F. Abouraddy, A. V. Sergienko, B. E. A. Saleh, and M. C. Teich, Phys. Rev. A 67, 062309 (2003).

[5] V. Giovannetti, L. Maccone, J. H. Shapiro, and F. N. C. Wong, Phys. Rev. Lett. 88, 183602 (2002).

[6] Z. D. Walton, M. C. Booth, A. V. Sergienko, B. E. A. Saleh, and M. C. Teich, Phys. Rev. A 67, 053810 (2003).

[7] M. Fiorentino, P. L. Voss, J. E. Sharping, and P. Kumar, IEEE Photonics Technol. Lett. 27, 491 (2002).

[8] X. Li, J. Chen, P. Voss, J. Sharping, and P. Kumar, Opt. Express 12, 3737 (2004).

[9] M. J. Fitch and J. D. Franson, Phys. Rev. A 65, 053809 (2002).

[10] C. K. Hong, Z. Y. Ou, and L. Mandel, Phys. Rev. Lett. 59, 2044 (1987).

[11] R. A. Campos, B. E. A. Saleh, and M. C. Teich, Phys. Rev. A
42, 4127 (1990).

[12] R. Erdmann, D. Branning, W. Grice, and I. A. Walmsley, Phys. Rev. A 62, 053810 (2000).

[13] V. Giovannetti, L. Maccone, J. H. Shapiro, and F. N. C. Wong, Phys. Rev. A 66, 043813 (2002).

[14] N. Imoto, H. A. Haus, and Y. Yamamoto, Phys. Rev. A 32, 2287 (1985).

[15] A. De Rossi and V. Berger, Phys. Rev. Lett. 88, 043901 (2002).

[16] D. Vakhshoori, R. J. Fischer, M. Hong, D. L. Sivco, G. J. Zydzik, G. N. S. Chu, and A. Y. Cho, Appl. Phys. Lett. 59, 896 (1991).

[17] F. De Martini, Phys. Rev. Lett. 81, 2842 (1998).

[18] A. M. Steinberg, P. G. Kwiat, and R. Y. Chiao, Phys. Rev. A 45, 6659 (1992).

[19] A. M. Steinberg, P. G. Kwiat, and R. Y. Chiao, Phys. Rev. Lett. 68, 2421 (1992).

[20] M. Tsang and D. Psaltis, Opt. Lett. 28, 1558 (2003). 